\title{
Health Care Delivery
}

National Cancer Institute

\section{Source}

National Cancer Institute. Health Care Delivery. NCI Thesaurus. Code C15219.

The provision of medical care. 